\section{Semantic priming in schizophrenia}

Sir: In their study on the effects of semantic priming on control subjects and subjects with schizophrenia (Weisbrod et al, 1998), the authors suggested that differences in performance relate to the presence or absence of thought disorder. However, the group of subjects defined as thoughtdisordered differed from other people with schizophrenia in that they had significantly less schooling, and also significantly higher Brief Psychiatric Rating Scale (BPRS) scores $(P=0.027$, not $P=0.27$ as they inadvertently stated). Thus, the differences they detect may well simply be manifestations of greater severity of illness, and may have nothing to do with thought disorder per se.

There are also grounds for concern regarding the instrument they have chosen to measure thought disorder. Subjects were defined as thought-disordered or not according to whether they scored higher than 3 on item 4 of the BPRS. This is the cut-off which distinguishes 'moderate' from 'mild' thought disorder, and might require the assessor to decide, on the basis of a single interview, whether the subject's degree of speech incomprehensibility is best described as: "occasional irrelevant statements, infrequent use of neologisms, or moderate loosening of associations", rather than "frequently vague, but the interview is able to progress smoothly; occasional loosening of associations". As Marder (1995) writes of this scale, "Without anchor points the definitions of the items can be vague and subject to different interpretations". Another disadvantage of the BPRS is that the reliance on a single item means that no attempt can be made to characterise different types of thought disorder, which may well be due to different underlying mechanisms rather than just due to aberrant association. Other instruments are far more satisfactory for this purpose. For example the Scale for Assessment of Thought, Language and Communication (Andreasen, 1979) has demonstrable reliability and uses multiple ratings to assess different aspects of thought disorder.

It is important that future studies are methodologically sound or else this one will join the many studies on thought disorder which have failed to be replicated.

Andreasen, N. C. (1979) Thought, language and communication disorders I, clinical assessment. definition of terms and evaluation of their reliability. Archives of General Psychiotry. 36. 1315-1321.

Marder, S. R. (1995) Psychiatric rating scales. In Comprehensive Textbook of Psychiatry (eds H. t. Kaplan \& B. J. Sadock). Baltimore, MD: Williams and Wilkins.
Welsbrod, M., Maler, S., Haris, S., et al (1998) Lateralised semantic and indirect semantic priming effects in people with schizophrenia. British journol of Psychiatry, 172, 142-146.

S. Bhandari, D. Curtis Tower Hamlets Healthcare NHS Trust. Department of Adult Psychiatry, The Royal London Hospital, London EI IBB

Author's reply: Bhandari \& Curtis correctly notice a misprint of a $P$ value. In the text we refer to the correct lower $P$ value and report significant differences between the BPRS scores of the two patient groups. This difference, however, was accounted for by the increased thought disorder ratings in the group with thought disorder. Hence, the general pathology of these patients is greater, but not their pathology as regards symptoms other than thought disorder (non-significant $t$-test). In our view, this state of affairs justifies the attribution of differences in dependent variables to the measure of thought disorder.

Moreover, if both groups had had the same BPRS sum score, one could have argued that - as thought disorder items reached high scores in the group with thought disorder - their remaining symptoms must have been lower. This would warrant the conclusion that the decreased general psychopathology of the patients with thought disorder is the cause of the increased priming effect. This conclusion, however, is ruled out by the higher general BPRS score in the thought-disordered group.

Generally speaking, the argument of non-specific differences is a serious one and has to be addressed in research on schizophrenia (cf. Chapman \& Chapman, 1978). However, if the results of an experimental procedure indicate that the worse-off patients perform comparatively better (i.e. show larger priming effects) then the argument is no longer valid. In other words, if the difference between patients with and without thought disorder were such that the patients with thought disorder had lower semantic priming, then the argument would hold. Since the patients with thought disorder have larger priming effects, these effects cannot be accounted for by a more pronounced general deficit.

Bhandari \& Curtis furthermore raise concerns about the way thought disorder was assessed in this study. In previous studies on priming effects in schizophrenia we had used other measures, among them the one they propose. However, these measures lump different kinds of symptoms together and are in no way indicators of the specific kind of loose associations we scrutinise in our work. Empirically, these measures came out worse than wellinformed clinical judgement when used to form patient subgroups.

In sum, the proof, here, is in the pudding. We have reliably measured semantic and indirect semantic priming effects in people with schizophrenia and compared subgroups with and without thought disorder. The effects and their specific lateralised pattern do not allow their attribution to a general deficit.

Finally, we want to emphasise that the increased semantic and indirect semantic priming effects in thought-disordered people with schizophrenia have been replicated several times in several languages using different methods (see Spitzer, 1997). We currently use event-related potentials in conjunction with semantic priming paradigms to clarify further the nature of formal thought disorder within the framework of cognitive neuroscience, and we aim at the exact characterisation of the involved dysfunctional cognitive processes in space and time.

Capman, L. J. \& Chapman, J. P. (1978) The measurement of differential deficit. Journal of Psychiotric Research, 14, 303-311.

Spitzer, M. (1997) A cognitive neuroscience view of schizophrenic thought disorder. Schizophrenia Bulletin. 23. 29-50.

M. Spitzer Universität Ulm, Psychiatrische Klinik, Leimgrubenweg 12-14, 89075 UIm. Germany

\section{Are first-rank symptoms encryption errors?}

Sir: Cognitive psychology has borrowed heavily from computer science, having incorporated notions such as memory 'encoding' and 'retrieval', articulatory 'loops' and visual 'scratch pads', and the 'global workspace' of consciousness, into accounts of human cognition. One problem facing cognitive neuropsychiatry is how cognitive accounts of psychotic symptoms might be implemented at the level of neurobiology. Accounts which treat subjective phenomena (quales) as analogous to 'software' properties, in contrast to neurological 'hardware', may perpetuate a form of dualism. How might quales and aberrant neurophysiology be reconciled?

I suggest one tentative reconciliation: that first-rank symptoms of schizophrenia 